Planta (Berl.) 94, 265-272 (1970)

(C) by Springer-Verlag 1970

\title{
Furfural Uptake by Neurospora Ascospores
}

\author{
Frederick I. Entures and Alfred S. Sussmax \\ Department of Botany, University of Michigan, Ann Arbor, Michigan
}

Received June 10, 1970

\begin{abstract}
Summary. Furfural uptake was studied with regard to possible mechanisms of inducing germination in ascospores. Uptake was found to involve a large, weakly bound reversible component and a small tightly bound irreversible component. Localization experiments indicate that almost all of the furfural removed from the media is bound to the spore wall. However, a small amount may penetrate into the cytoplasm. The results so far suggest that furfural induces germination by solubilizing or activating a bound or compartmentalized enzyme(s) on the cell membrane or other diffusion barrier of the cell.
\end{abstract}

\section{Introduction}

The activation or breaking of dormancy in Neurospora ascospores by either heat shock (Shear et al., 1927) or furfural treatment (Emerson, 1948) has been known for many years. However, the exact molecular mechanism of either mode of activation is not completely understood.

Previously, furfural activation has been studied with regard to the receptiveness of ascospores to furfural by Emerson (1952) and the furfural induced respiratory responses of ascospores (Sussman, 1953). The present authors have examined furfural activation by investigating the metabolism of furfural by ascospores. These studies have shown that there are two aspects to furfural metabolism: A. The conversion of furfural to furoic acid and furfuryl alcohol; and B. The uptake of furfural.

In addition, the metabolic changes occurring during activation has been examined. The results of these investigations suggested that immediately after treatment the spores undergo a rapid glycolysis and alcohol fermentation, and that a functional aerobic respiratory system developed during the germination process (Sussman et al., 1956). Also, dormant ascospores have been shown to metabolize lipids, whereas activated spores also utilize trehalose, the main storage carbohydrate in the spore (Lingappa et al., 1959). Presumably, the glucose derived from trehalose could account for the rapid glycolysis. A trehalose hydrolyzing enzyme (trehalase) was found in both dormant and activated spores so its de novo synthesis is not a prerequisite for activation (Hill et al., 1964).

Recent studies by the authors have shown that immediately upon activation by either heat or furfural, certain metabolic intermediate 
concentrations change. Of particular interest are those reactions involving G-6-P and F-6-P formation and trehalose utilization.

With knowledge of these changes occurring within the ascospores, it was important to investigate furfural uptake more closely with particular regard to possible mechanisms whereby furfural could activate the spore.

\section{Materials and Methods \\ Preparation of Ascospores}

Ascospores were grown and harvested by techniques described elsewhere (Goddard, 1935) with the exception of the EDTA treatment which was lengthened from 24 hours to 7 days.

The spores are designated as follows: Dormant ascospores are those which have not received any treatment beyond the EDTA wash. Heat-sensitized spores are dormant spores which have received a heat treatment $\left(45^{\circ} \mathrm{C}\right.$ for 30 minutes) (Sussman, 1954) which causes them to respond maximally to furfural. Heatsensitization alone will-not induce germination. Furfural-activated spores are those which have been heat-sensitized and then immediately incubated in $1 \times 10^{-3} \mathrm{M}$ furfural at room temperature. Only a few minutes in contact with furfural is required to activate the spores. However, unless otherwise mentioned, the ascospores were continuously incubated in furfural. Furfural-activated ascospores germinate some 3 hours after the addition of furfural.

\section{Preparation of Furfural}

In most experiments it was necessary to use $\mathrm{C}^{14}$-furfural for the measurement of furfural uptake. Accordingly, $\mathrm{C}^{14}$ furfural was synthesized by an acid dehydration of pentoses and pentosans according to the following procedure: Powdered $\mathrm{C}^{14} \mathrm{O}_{2}$ grown snapdragon stems $(2 \mathrm{~g})$ were added to $100 \mathrm{ml}$ of $12 \% \mathrm{HCl}$. The mixture was boiled vigorously for 90 minutes after which the distillate was neutralized with $\mathrm{NaOH}$. This neutralized solution was distilled to remove the $\mathrm{NaCl}$, and then applied to an Amberlite IRA 400 column $(1 \mathrm{~cm} \times 30 \mathrm{~cm})$ in the bisulfite form. The column was then washed with $100 \mathrm{ml}$ of $80^{\circ} \mathrm{C}$ water to remove ketones before furfural was diluted with sodium carbonate $(0.1 \mathrm{M})$. The tubes containing radioactivity were stored frozen and were thawed and acidified with $\mathrm{HCl}$ to $\mathrm{pH} 1.0$ to break the bisulfite furfural complex just prior to use. Furfural was then distilled microdistillation apparatus. The distillate was neutralized and used within one or two days after preparation.

The purity of the C14-furfural was determined by its absorbtion spectrum in the ultraviolet range and by chromatography of the 2-4 dinitrophenylhydrazone derivative in 2 solvent systems (Schmitt et al., 1956).

\section{Assay of Furfural}

Measurement of furfural concentrations utilized linear absorption at the absorption maximum of $277 \mathrm{~nm}$, and in addition for $\mathrm{C}^{14}$ labeled furfural, radioactivity measurements were made. The samples $(0.5 \mathrm{ml})$ used for radioactivity determinations were dissolved in $15 \mathrm{ml}$ of scintillation solution (Bruno et al., 1961) and cooled thoroughly before counting. Quench correction was made by internal standardization.

In all of the experiments the incubation media were used as samples for furfural measurements by removing an aliquot of spores and media and then separating the spores from the media by filtration. 


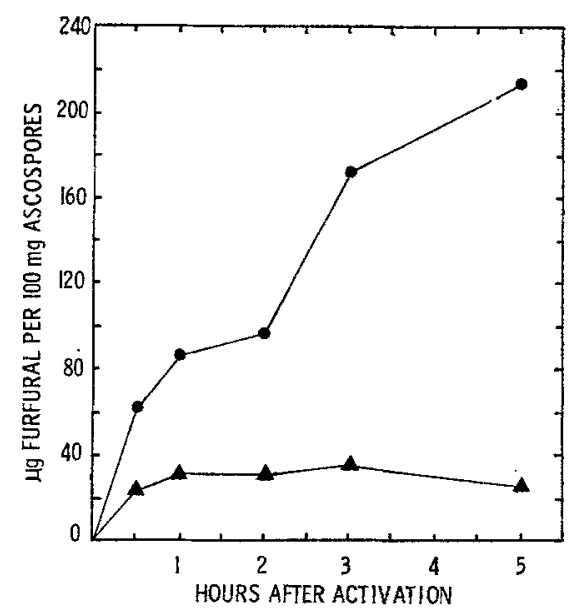

Fig. 1. Furfural uptake and conversion as determined by radioactivity measurements (triangles) and UV absorption (circles) per $100 \mathrm{mg}$ untreated ascospores at room temperature. The spores were incubated in $2 \times 10^{-3} \mathrm{MI}$ furfural at a ratio of $100 \mathrm{mg}$ spores per $1 \mathrm{ml}$ of furfural solution

\section{Results}

The incubation of ascospores in labeled furfural results in two separate processes whereby furfural is consumed: uptake and conversion to other compounds. These processes are seen clearly in Fig. 1. Ultraviolet light absorption measurements show a rapid decrease in furfural while the radioactivity measurements show a very different pattern. These measurements may be correlated by assuming that the UV measurements monitor the conversion of furfural to compounds which do not absorb at $277 \mathrm{~nm}$ but which remain in the medium; while the radioactivity measurements monitor uptake by measuring the disappearance of label from the medium. This assumption was varified by identification of furoic acid and furfuryl alcohol as conversion products and is discussed in detail elsewhere.

As a control for the effects of possible contamination, ascospores were shaken in $0.1 \mathrm{~N} \mathrm{HCl}$ for 30 minutes before being washed free of acid and incubated in furfural. Acid treatment has no detrimental effect on ascospores. Furfural uptake by these spores was identical to that of non-acid treated spores (Fig. 1) and consequently is not shown. Also contaminants were looked for by direct plating of aliquots of the activation medium which always resulted in very low bacterial and fungal colony counts. Thus, it appears that uptake is a property of the ascospores and is not due to contaminants. 


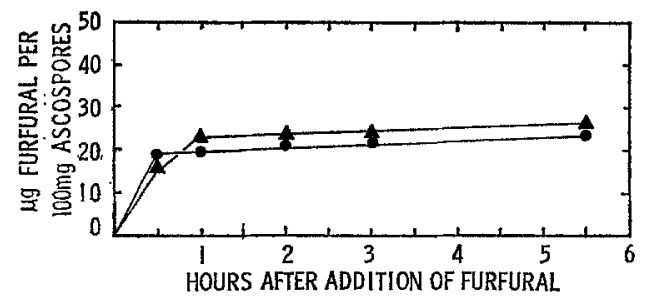

Fig. 2. Furfural uptake as determined by radioactivity measurements (triangles) and UV absorption (circles) per $100 \mathrm{mg}$ heat "killed" ascospores at room temperature

\section{Reversible and Irreversible Adsorption of Furfural}

The kenetics of furfural uptake with either group of spores were similar to an adsorption isotherm. This suggests that furfural, like many compounds, may bind to the cell surface (Lowry et al., 1957). To test whether living cells were necessary for uptake, heat killed (autoclaved dry for 20 minutes) ascospores were incubated in furfural with only slightly less uptake than living spores (Fig. 2).

This slight difference between living and killed spores indicates that most of the furfural is taken up by an adsorption or "free space like" compartment, and comparatively little, if any, is due to transport into the cell. The nature of the passive uptake was further investigated by incubating several lots of spores briefly in $\mathrm{C}^{14}$-furfural and determining their uptake before reincubating each spore lot in a second solution containing either unlabeled furfural, formaldehyde, or distilled water. If only an adsorption or "free space" uptake is involved, then it should be possible to exchange the $\mathrm{C}^{14}$-furfural for the unlabeled material in the media. The results of these experiments (Fig. 3) show that approximately $75 \%$ of the $\mathrm{C}^{14}$-furfural taken up by the ascospores is lost under all experiment conditions. Uptake thus appears to involve a large reversible component and a smaller absorption or irreversible component.

\section{Uptake Localization}

Uptake was further investigated in a series of experiments designed to aid in localizing the area(s) of the cell into which the material was taken up. If the reversible component is due to adsorbtion to the spore wall, then removal of the outer wall layers (Lowry et al., 1957) might reduce uptake. The outer wall layers were removed by soaking spores in a $20 \%$ Clorox solution for 2-60 minutes and then washing with distilled water. Although complete wall removal was not accomplished by this technique, all of the walls remaining were swollen. After heat-sensitization, these spores were incubated in furfural and monitored by UV absorption. While such measurement normally indicates only furfural conversion, the 


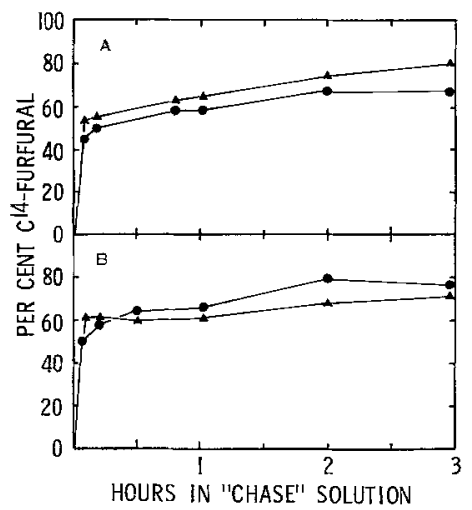

Fig. 3. Exchange of $\mathrm{C}^{14}$-furfural for unlabeled exogenous material. After a $15 \mathrm{~min}$ incubation in labeled furfural, ascospores were removed and aliquots were placed in a known amount of the second medium. The amount of $\mathrm{C}^{14}$-furfural reappearing in the second medium was calculated on the basis of initial specific activity. The initial concentration of furfural $\left(1 \times 10^{-3} \mathrm{M}\right)$ was $100.8 \mu \mathrm{g}$ per $100 \mathrm{mg}$ ascospores, and after $15 \mathrm{~min}$ the spores took up $29.2 \mu \mathrm{g}$ per $100 \mathrm{mg}$ spores. The average amount of labeled furfural remaining in the spores at the end of the experiment was $7.6 \mu \mathrm{g}$ per $100 \mathrm{mg}$ ascospores. In graph $\mathrm{A}$, circles represent exchange for $1 \times 10^{-1} \mathrm{M}$ furfural and triangles represent exchange for $1 \times 10^{-2} \mathrm{M}$ furfural. In graph $\mathrm{B}$, circles represent exchange for $10 \%$ formaldehyde and triangles represent exchange in distilled water

results obtained in these experiments (Fig.6,p. 262, this issue) for the first 60 minutes after furfural addition, more closely resemble those expected for uptake rather than conversion. These results are interpreted to mean that the removal of some of the spore walls and the swelling of the remaining walls results in approximately a $50 \%$ reduction in uptake and essentially, a complete inhibition of conversion. Although germination was decreased by the Clorox treatment, spore germination did occur ranging from $14 \%$ for the 60 minute Clorox sample to $46 \%$ for the 5 minute sample.

In addition to experiments using a surface treatment such as Clorox, the site of uptake was investigated using fractionated ascospores. Heatsensitized ascospores were homogenized in the syringe spore grinder (Eilers et al., 1965) at $4^{\circ} \mathrm{C}$, fractionated by centrifugation, and then incubated in furfural. As the data (Fig. 4) show, the wall fraction adsorbs a large proportion of the furfural present. Although these measurements were also made by UV absorption, the interaction of furfural and the wall fraction in one of uptake and not conversion since the furfural is recovered from the walls as they age. The intermediate fraction has some activity which could either be adsorption by the wall material present or a small degree of conversion. The soluble fraction shows no activity. More furfural is adsorbed by the homogenized walls than by an 


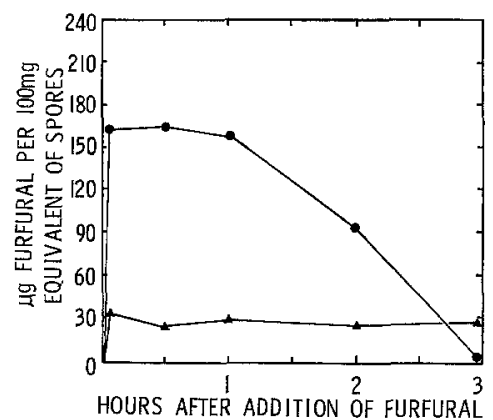

Fig. 4. Furfural conversion and uptake in cell-free extracts of ascospores. The spores were homoginized, centrifuged, and separated into a wall fraction (circles) $(0-1,000$ $\times \mathrm{g})$; an intermediate fraction (triangles), $(1,000-15,000 \times \mathrm{g})$; and a supernatant fraction (over 15,000 $\times \mathrm{g}$ ) that did not show any activity. The ascospores incubated in $211.2 \mu \mathrm{g}$ furfural $\left(2 \times 10^{-3} \mathrm{M}\right)$ per $100 \mathrm{mg}$ equivalent of spores. All concentrations were measured by UV absorption

Table. The distribution of furfural in ascospores

$200 \mathrm{mg}$ ascospores were activated in $\mathrm{C}^{14}$-furfural for 10 minutes before the excess furfural was removed. The spores were then washed in three 5-ml portions of water to remove the nonabsorbed furfural. After washing, the spores were ground in the syringe spore grinder and separated by centrifugation at $10,000 \times \mathrm{g}$ into a wall and a supernatant fraction. The supernatant fraction was assayed directly, while the wall fraction was oxidized to $\mathrm{C}^{14} \mathrm{O}_{2}$ by conc. sulfuruc acid before its activity was assayed

\begin{tabular}{lccc}
\hline $\mathrm{C}^{14}$-furfural concentration expressed as & $\mu g$ & CPM & $\begin{array}{c}\% \text { of } \\
\text { total } \\
\text { uptake }\end{array}$ \\
\hline C $^{14}$-furfural taken up by the ascospores & 55 & 240,264 & 100 \\
$\begin{array}{l}\text { C }^{14} \text {-furfural removed from the ascospore } \\
\text { in three washes }\end{array}$ & 43 & 187,290 & 77.9 \\
$\mathrm{C}^{14}$-furfural remaining in the ascospore & 12 & 52,974 & $\begin{array}{c}22.1 \\
(100)\end{array}$ \\
$\begin{array}{l}\text { C }^{14} \text {-furfural found in the supernatant } \\
\text { fraction }\end{array}$ & 1.2 & 5,213 & 9.8 \\
$\begin{array}{l}C^{14} \text {-furfural found in the wall fraction } \\
\text { upon combustion of the wall to } \mathrm{C}^{14} \mathrm{O}_{2}\end{array}$ & 9.4 & 36,489 & 78.5 \\
\begin{tabular}{l} 
Per cent recovery \\
\hline
\end{tabular} & & & 88.3 \\
\hline
\end{tabular}

equivalent weight of whole spores, probably due to an increase in surface area.

While these experiments indicate that the spore wall is a major site of furfural adsorption, they do not discriminate between the reversible and irreversible aspects of the process. To determine if the irreversible 
aspect represented binding to the spore wall or transport into the soluble portion of the spore, intact spores were heat-sensitized and incubated in $\mathrm{C}^{14}$-furfural for 10 minutes. The exchangeable $\mathrm{C}^{14}$-furfural was removed with repeated washing for 90 minutes. The washed spores now containing only the nonexchangeable furfural were homogenized and separated by centrifugation into a wall and a supernatant fraction. About $10 \%$ of the total nonexchangeable $\mathrm{C}^{14}$-furfural (Table) was recovered in the supernatant fraction. The majority $(78 \%)$ of the unaccounted for $\mathrm{C}^{14}$-furfural was recovered from the wall fraction when it was oxidized to $\mathrm{C}^{14} \mathrm{O}_{2}$ with concentrated sulfuric acid. These data indicate that most of the nonexchangeable furfural is bound to some part of the spores exterior, although a small amount may enter the interior of the spore.

\section{Discussion}

In attempting to understand the action of furfural in breaking dormancy in ascospores, one must try to correlate internal metabolic change and the interaction of furfural and the ascospore.

Of the two processes one must consider, conversion and uptake, furfural conversion does not appear to the implicated in activation for several reasons: a) the internal metabolic change occurs within 2 or so minutes and conversion is much slower; b) the first conversion product, furoic acid is completely ineffective in spore activation ; and c) conversion can be inhibited without preventing activation. Thus, the uptake of furfural as furfural is responsible for ascospore activation.

Within uptake itself there are two components to consider: reversible and irreversible uptake. While it has not been possible to selectively inhibit one or the other and note the effect, the ease of removing the reversible component indicates it is due to a weak adsorption or to a free space uptake within the heary spore wall; and thus apears not to be involved meaningfully in activation. Consequently, the furfural irreversibly bound by the spores ( $25 \%$ of the total uptake or $7.6 \mu \mathrm{g}$ per $100 \mathrm{mg}$ spores) is apparently responsible for activation.

From the localization experiments it can be concluded that most of the bound furfural is bound to some part of the spore wall. The remainder, that is found in the soluble fraction, may represent penetration into the cytoplasm of the spore or it may be a resultant of the homogenization process. This value was very consistant from experiment to experiment so it may well represent penetration. However, our failure to conclusively demonstrate furfural penetration into the cytoplasm of the spore suggests that furfural exerts its effect on or outside of any diffusion barriers in the spore.

The metabolic changes induced by activation occur within minutes after treatment so it is felt that spore activation involves the activation of existing enzymes. This being the case, furfural could activate by 
solubilizing an enzyme(s) bound or compartmentalized on the cell membrane or other diffusion barrier. Alternatively, it may directly affect an inactive but permanently bound enzyme(s).

The enzymes thought to be involved in activation based on metabolic intermediate data are those involved in trehalose degregation and G-6-P and F-6-P formation. The effect of furfural must now be tested in vitro on these enzymes. At present, work is in this direction and soon it may be possible to demonstrate the specific action of furfural in ascospore activation.

This paper is part of a dissertation submitted to the Rackham Graduate School of the University of Michigan in partial fulfillment of the requirement for the Doctor of Philosophy degree in Botany by Frederick I. Eilers.

\title{
References
}

Bruno, G. A., Christian, J. E.: Determination of Carbon-14 in aqueous bicarbonate solutions by liquid scintillation counting techniques. Anal. Chem. 33, 1216-1218 (1961).

Eilers, F. E., Fischer, G.: An efficient grinder for ascospores and mycelium of Neurospora. Neuro. Newsl. No 8 (1965).

Emerson, M. R.: Some physiological characteristics of ascospore activation in Neurospora crassa. Plant Physiol. 29, 418 - 428 (1952).

- Chemical activation of ascospore germination in Neurospora crassa. J. Bact. 55, 327-330 (1948).

Goddard, D. R.: The reversible heat activation inducing germination and increased respiration in the ascospores of Neurospora tetrasperma. J. gen. Physiol. 19, $45-69$ (1935).

Hill, E. P., Sussman, A. S.: Development of trehalase and invertase activity in Neurospora. J. Bact. 86(6), 1556-1566 (1964).

Lingappa, B. T., Sussman, A. S.: Endogenous substrates of dormant, activated and germinating ascospores of Neurospora tetrasperma. Plant Physiol. 34(4), 466$472(1959)$.

Lowry, R. J., Sussman, A. S.: Wall structure of ascospores of Neurospora tetrasperma. Amer. J. Bot. 45 (5), 397-403 (1958).

- - Boventer, B. von: Physiology of the cell surface of Neurospora ascospores. III. Distinction between the adsorptive and entrance phases of cation uptake. Mycologia 49(5), 609-622 (1957).

Schmitt, W. J., Noreconi, E. J., Conner, W. F. O.: Paper chromatography of nitrophenylhydrozones of mono and dicarbonyl compounds. Anal. Chem. 28, 249 (1956).

Shear, C. L., Dodge, B. O.: Life histories and heterothallism of the red bread-mold fungi of the Molinia sitophila group. J. agric. Res. 34, 1019-1042 (1927).

Sussman, A. S., Distler, J. R., Krakow, J. S.: Metabolic aspects of Neurospora activation and germination. Plant Physiol. 31 (2), 126-135 (1956).

- The effect of furfural upon the germination and respiration of ascospores of Neurospora tetrasperma. Amer. J. Bot. 40(6), 401-404 (1953).

\author{
Frederick I. Eilers \\ Department of Botany and Bacteriology \\ University of South Florida \\ Tampa, Florida 33620, U.S.A
}

